\title{
An instrumented minimally invasive surgical tool: Design and calibration
}

\author{
Philip R. Roan ${ }^{\mathrm{a}, *}$, Andrew S. Wright ${ }^{\mathrm{a}}$, Thomas S. Lendvay ${ }^{\mathrm{a}, \mathrm{b}}$, Mika N. Sinanan ${ }^{\mathrm{a}}$ and Blake Hannaford ${ }^{\mathrm{a}}$ \\ ${ }^{a}$ University of Washington, Seattle, WA, USA \\ ${ }^{\mathrm{b}}$ Seattle Childen's Hospital, Seattle, WA, USA
}

\begin{abstract}
Minimally invasive surgical procedures have improved the standard of patient care by reducing recovery time, chance of infection, and scarring. A recent review estimates that leaks occur in 3\% to $6 \%$ of bowel anastomoses, resulting in "increased morbidity and mortality and adversely [affecting] length of stay, cost, and cancer recurrence" [23]. Many of these leaks are caused by poor handling and ischemic tissue.

Detecting a change in temperature can indicate ischemic tissue. The optical absorption spectrum of a tissue can be used to detect tissue oxygen concentration and tissue ischemia. The electrical impedance of tissue changes as ischemia progresses.

This article describes the development of a minimally invasive surgical tool with integrated sensors for replicating ischemia detection measurements during routine manipulation of the tissue. To be useful, this tool should be feasible for use in a real operating room, providing real-time feedback and diagnosis to the surgeon. The design of the tool and choice of the sensors leverages existing work in physiological measurements and surgical tool design.

The tool includes a thermistor for measuring the temperature, four LEDs and a photodiode for measuring local optical absorption, and four electrodes for measuring the electrical impedance. The sensors are located on a $7 \mathrm{~mm}$ square sensor head, which is mounted to a minimally invasive grasper. A strain gauge and optical encoder monitor the applied force and position of the tool, and a motor controls both. This allows the tool to control the tool-tissue interface. Sensor accuracy has been validated through calibration.
\end{abstract}

Keywords: Ischemia detection, optical spectroscopy, electrical impedance, surgical tool design

\section{Introduction}

Minimally invasive surgical procedures have improved the standard of patient care by reducing recovery time, chance of infection, and scarring. Robotic surgery has further increased the standard of care by reducing the difficulty of procedures and enabling other procedures that were previously impossible, such as microsurgery. However, these new techniques and tools place the surgeon farther away from the surgery site resulting in reduced tactile feedback, which is use-

${ }^{*}$ Corresponding author. E-mail: proan@uw.edu. ful for diagnosis and proper manipulation of tissue [35].

Improper tissue handling during surgery can cause adhesion, bleeding, perforation, and it can restrict the flow of blood to the tissue [10]. It is hypothesized that instrumented surgical tools can reduce the incidence and severity of tissue damage during minimally invasive surgeries, particularly for bowel anastamoses.

A recent review estimates that leaks occur in $3 \%$ to $6 \%$ of bowel anastomoses, resulting in "increased morbidity and mortality and adversely (affecting) length of stay, cost, and cancer recurrence" [23]. Poor handling and the resulting ischemic tissue are cited as the root of many failures. 
Currently the surgeon visually inspects for ischemic tissue, but this method is not always reliable [3]. Ischemia can be detected by measuring tissue temperature, optical absorption, or electrical impedance. There is a large body of work relating these individual measurements to tissue ischemia, but they all rely on fixed probes or large sensors.

This article describes the development of a minimally invasive surgical tool with integrated sensors for replicating ischemia detection measurements during routine manipulation of the tissue. To be useful, this tool should be feasible for use in a real operating room, providing real-time feedback and diagnosis to the surgeon. Additionally, the hardware should be easily transported to the operating room, and the tool and sensors should be robust enough to be sterilized or cheap enough to be disposed after an operation.

This article will briefly discuss some medical benefits provided by an instrumented surgical tool, the existing sensing modalities for detecting ischemia, an overview of the tool design, and calibration equations and data for the tool.

\subsection{Medical need}

As mentioned above, 3-6\% of bowel anastomoses leak and require an additional surgery to repair. Intraoperative risk factors include long operations and suboptimal blood supply. Tissue ischemia at the site of the anastomosis is frequently cited as the cause for leak [23].

Ischemia is the restriction of arterial blood supply to a level below what the tissue demands resulting in the shortage of oxygen and nutrients and the build up of metabolic waste products [21]. Ischemia is closely related to hypoxia, which is the lack of oxygen in a tissue. As ischemia progresses, metabolic waste products can build up, lowering tissue $\mathrm{pH}$, and the eventual cellular death, or infarction, causes tissue damage [34].

For the surgeon, it is very difficult to detect hypoxia with the naked eye [22]. This makes the determination of viable tissue for anastomosis difficult without sensors for ischemia and hypoxia detection. If ischemia is detected early, it may be totally reversible. Should bowel ischemia go undetected, the resulting infarction can have fatal consequences [36]. The mortality rate from late or missed diagnoses of intestinal infarction is $60 \%$ to $90 \%$ [34].

Timely diagnosis of ischemia and intervention are important for reducing the rate of bowel leaks and hospital readmissions thereby improving patient outcomes. Integrating sensors into a surgical tool will allow for the detection of ischemia while the tissue is being manipulated.

\section{Background}

The design of the tool and choice of the sensors leverages existing work in physiological measurements and surgical tool design. There is a significant body of work related to the detection of ischemia and hypoxia. The most accurate techniques involve directly measuring the percentage of gases like oxygen and carbon dioxide in the blood [41]. However, these techniques are also the most invasive and intensive. Less invasive techniques have been developed and are based on sensing tissue temperature, optical absorption spectra, or electrical impedance spectra. These modalities will be discussed followed by methods of combining the data from multiple modalities.

\subsection{Temperature}

Detecting a change in temperature can indicate ischemic tissue and can be useful in determining viable bowel tissue for use in anastomoses [7]. Thermal imaging has been used to detect ischemia in bowel after 5 minutes of warm ischemia time. Warm ischemia time is the length of time that a tissue remains at body temperatures after having its blood supply removed. In the same tests, surgeons were unable to differentiate vascularized and devascularized bowel using standard visual inspection techniques [3].

Tissue temperature can be sensed directly with thermometers, thermocouples, or thermistors; it can also be sensed indirectly by measuring infrared radiation.

\subsection{Optical absorption}

It is well established that the optical properties of tissue can be used to estimate the level of oxygen of the blood in tissue [41]. Oxygenated and deoxygenated hemoglobin have different optical absorption spectra. Both are different from carboxyhemoglobin (HbCO), which is hemoglobin bound to carbon monoxide instead of oxygen. The absorptivities of oxyhemoglobin $\left(\mathrm{HbO}_{2}\right)$ and deoxyhemoglobin $(\mathrm{Hb})$ are shown from $250 \mathrm{~nm}$ (ultraviolet) to $1000 \mathrm{~nm}$ (near infrared) in Fig. 1. The concentration of hemoglobin 


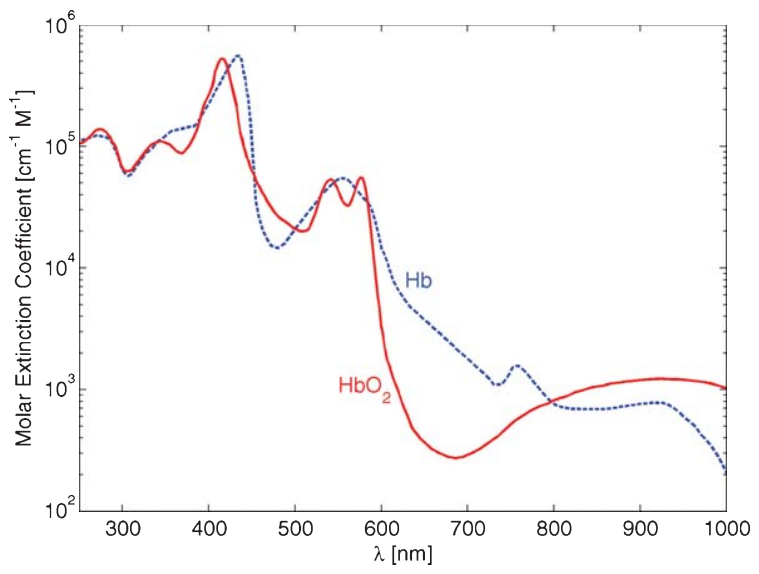

Fig. 1. Extinction coefficents (absortivities) of oxyhemoglobin $\left(\mathrm{HbO}_{2}\right)$ and deoxyhemoglobin $(\mathrm{Hb})$. Data from [30].

can be estimated by measuring the light transmitted through the blood and using Beer's law:

$$
A=e l C
$$

where $A$ is the absorbance, $e$ is the extinction coefficient (Fig. 1), $l$ is the path length, and $C$ is the concentration of the absorbing substance. Absorbance is related to the percentage of light transmitted through the tissue, $\% \mathrm{~T}$, by

$$
A=\log _{10} \frac{100}{\% T}
$$

The absorbance of the tissue can also be measured from the light reflected back by the tissue [29, 31].

Beer's law does not account for light scattering in the tissue, and so calibration curves are required. These calibration curves are compiled from healthy volunteers, and often have very few data points at oxygen saturation levels below 80\% [38]. Efforts have been made to use a better optical model than Beer's law for increased accuracy, especially at low oxygen saturations [45].

Oxygen saturation of blood, $\mathrm{So}_{2}$, is the percentage of hemoglobin molecules that are bound to oxygen, whereas the partial pressure of oxygen in blood, $\mathrm{Po}_{2}$, is related to the amount of oxygen gas dissolved in the plasma. Normally $98 \%$ of the oxygen transported by the blood is bound to hemoglobin and $2 \%$ is dissolved in the plasma, but this percentage is temperature and $\mathrm{pH}$ dependent [41]. The ratio of the concentration of oxyhemoglobin to the total concentration of hemoglobin gives $\mathrm{So}_{2}$ :

$$
\mathrm{So}_{2}=\frac{\left[\mathrm{HbO}_{2}\right]}{\left[\mathrm{Hb}+\mathrm{HbO}_{2}\right]}
$$

It is often assumed that the there are only two species $\left(\mathrm{HbO}_{2}\right.$ and $\left.\mathrm{Hb}\right)$ in the blood so the absorbance can be computed using superposition:

$$
A=l \cdot\left(e_{o}\left[\mathrm{HbO}_{2}\right]+e_{r}[\mathrm{Hb}]\right)
$$

where $e_{o}$ and $e_{r}$ are the extinction coefficients of $\mathrm{HbO}_{2}$ and $\mathrm{Hb}$, respectively. The path length dependance can be eliminated by measuring the absorbance at two or more wavelengths.

The two wavelengths often used in oximetry are $660 \mathrm{~nm}$ (red) and $940 \mathrm{~nm}$ or $805 \mathrm{~nm}$ (infrared) because the greatest difference in extinction coefficients occurs at $660 \mathrm{~nm}$ and the smallest difference at $805 \mathrm{~nm}$, the isosbestic wavelength ${ }^{1}$ [41].

The simplifying assumption used to derive Equation 4 is generally valid when measuring only arterial blood. When measuring the oxygen in an organ, the tissues and venous blood are additional absorbers which effectively add terms to Equation 4. This significantly complicates the calculations because the extinction coefficients for the additional absorbers may be time varying, may not be homogenous, and are potentially unknown.

The most common solution to this problem is to compare absorbance measurements at systole and diastole. The difference in absorbance is assumed to be due to arterial blood alone, and then Equation 4 can be applied.

If a pulse cannot be found, a different method of solving for the oxygen saturation must be used. By using more than two wavelengths of light, the extinction coefficients and concentrations of additional absorbers can be approximated. An early oximeter produced by Hewlett-Packard used eight wavelengths [41].

Most methods for measuring the optical absorption properties of tissues involve contact between the tissue and the sensor $[1,16]$. Contact between the tissue and the sensor is often necessary to reduce the influence of background radiation and maintain a uniform contact interface as there can be significant motion artifacts from moving sensors or tissues or both [39, 42]. However, the nature of this contact will affect the measurement by potentially creating local tissue deformation or increasing the tissue temperature. Thus it is important

\footnotetext{
1 The isosbestic point is where the extinction coefficients are the same for $\mathrm{HbO}_{2}$ and $\mathrm{Hb}$.
} 


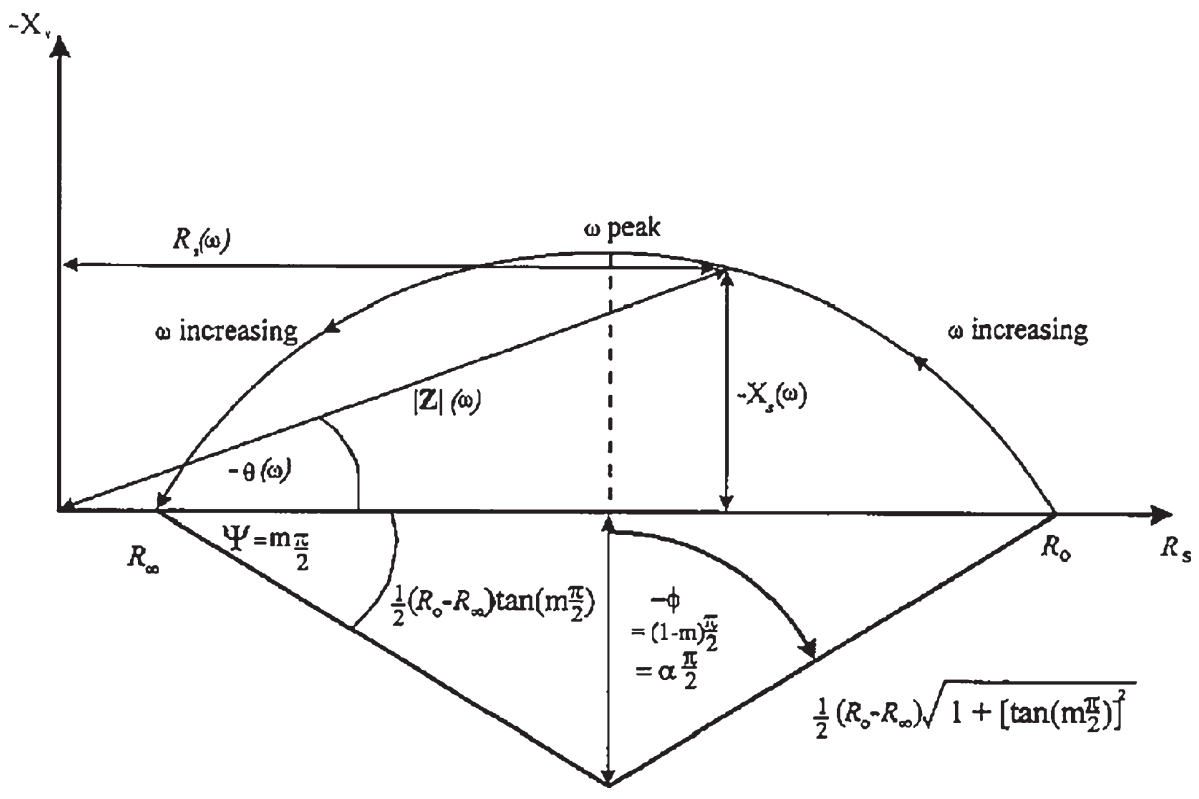

Fig. 2. A diagram of a complex impedance locus for a typical biological tissue. Reproduced from [27].

to also measure the pressure applied by the sensor and the local heating caused by the sensor [2].

\subsection{Electrical impedance}

A typical tissue impedance can be closely approximated by Cole's equation [27]:

$$
Z=R_{\infty}+\frac{R_{0}-R_{\infty}}{1+\left(j \omega / \omega_{o}\right)}
$$

where $Z$ is the impedance, $R_{\infty}$ is the resistance at high frequencies, $R_{0}$ is the impedance at low frequencies, $\omega_{o}$ is the frequency where the impedance has the greatest reactance, and $\alpha$ is a dimensionless constant between zero and one that describes the depression of the arc below the real axis. A plot of this equation in the complex impedance plane is shown in Fig. 2; it should be noted that the plot axes are the positive real component and the negative imaginary component. In ischemic tissue, resistance at lower frequencies increases with ischemia duration while resistance at high frequencies is independent of ischemia [33].

The measured phase angle of biological tissues is constant over a wide range of frequencies and is modeled with a constant-phase element [27]. A standard circuit model for biological tissues is shown in Fig. 3.

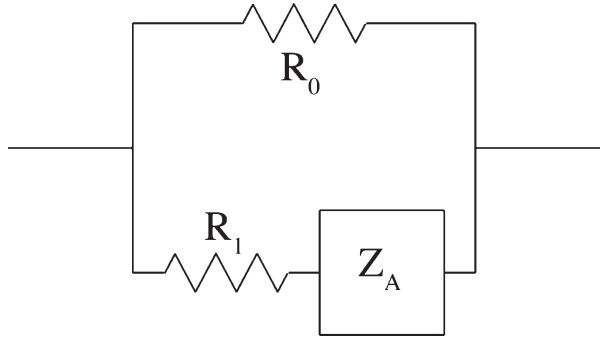

Fig. 3. A typical biological tissue circuit model. $Z_{A}$ is a constantphase element, and $R_{1}$ in parallel with $R_{0}$ equal $R_{8}$.

An equation for the constant-phase element, $Z_{A}$, can be derived from Equation 5.

Tissue impedance is often measured by means of a linear array of four electrodes. The drive electrodes (outer pair) apply a constant magnitude current and the sense electrodes (inner pair) sense the resulting voltage difference $[37,40]$. The impedance is measured over a range of frequencies, though typically between $100 \mathrm{~Hz}$ and $1 \mathrm{MHz}[8,19,32]$.

There is a strong body of work that measures the changes in impedance of ischemic tissue over time. This is due to cell swelling, closing intercellular gap junctions, and the accumulation of metabolic products [18]. Figure 4 shows the change in impedance of a rabbit leg muscle as ischemia progresses. Additionally, 


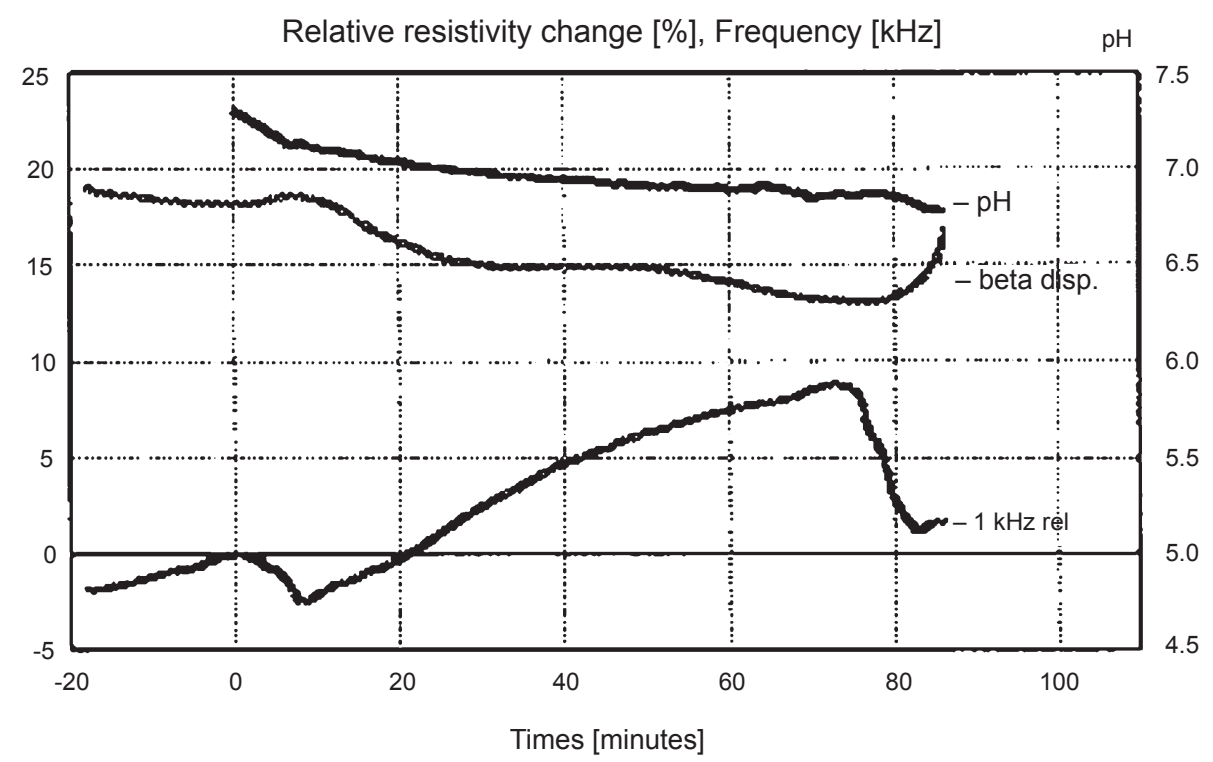

Fig. 4. Typical measured resistivity, $\mathrm{pH}$ and beta dispersion over time after the onset of ischemia. Beta dispersion is a measure of the change of capacitance. Tissue is the isolated tibialis anterior muscle of a rabbit hind leg. The occlusion of the artery was removed at 72 minutes. Reproduced from [24]. Additional plots showing the relationship between impedance and ischemia can be found in [18].

the impedance of a wide variety of tissues, including muscle, liver, spleen, kidney, and intestine, have been measured and tabulated. The results are given as in terms of the tissue model parameters described in Equation 5 [8, 32]. These models can be used to differentiate tissues.

\subsection{Force and displacement}

Minimally invasive surgery results in a reduction of force and tactile feedback to the surgeon [28, 35]. Work has been done to restore the sense of touch to the surgeon by adding position, force, and tactile sensors to surgical tools $[15,35]$. Safe handling of tissues during surgery requires manipulating tissues in a way that is secure and not damaging [20].

Mechanically compressing a tissue, such as during a grasping procedure, causes tissue damage that is a function of the applied compressive stress [11, 12]. For computer controlled tools, safe handling of tissue can be implemented using the knowledge of acceptable damage levels, a model of the mechanical properties of the tissue, and the force applied by the tool [43, 44].

Incorporating force and position sensing will allow for the sensor-tissue contact interface to be controlled while also controlling the amount of damage to the tissue from grasp events.

\subsection{Multiple sensor data fusion}

Collecting data from multiple sensors has the potential to increase the reliability of measurements. Other typical goals for combining sensor data are to reduce the dimensionality of the data or to increase the signalto-noise ratio of the data.

The tissue property and ischemia measurements discussed above were made with specialized probes. Miniaturizing these sensors and fitting them on to the end of a tool for minimally invasive surgery can cause difficulties with noise and other disturbances. However, it should be possible to achieve similar, or possibly better, measurements by incorporating multiple sensing modalities and fusing the data.

Additionally, measurements from each modality are not completely independent. For example, LEDs placed on the surface of the tissue can heat the tissue [2]. Surgical graspers and retractors with integrated pulse oximetry and force sensing have been built at Johns Hopkins University. Preliminary results show a change in the ratio in the oximetry signals when force is applied to the tissue [14].

\section{Design}

The tool is built on the Motorized Endoscopic Grasper (MEG) developed in the BioRobotics Lab 


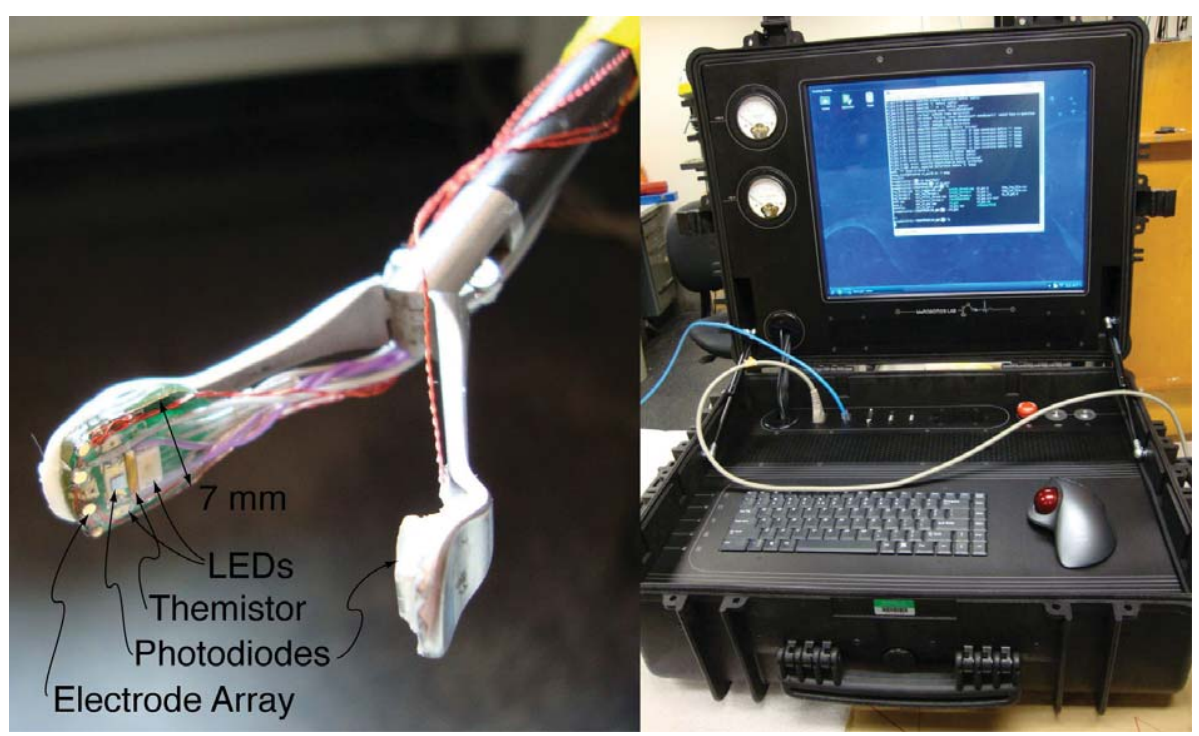

Fig. 5. (Left) A photograph of the sensor heads mounted to the grasper. (Right) A photograph of the control box running a real-time enabled version of Linux.

at the University of Washington $[4,6]$, which uses a standard Babcock grasper (\#33510 BL, Karl Storz). The Babcock grasper ensures nearly uniform pressure across the contact surfaces of the jaws. Grasp force and jaw position are sensed and controlled, thereby controlling the tool-tissue interface.

The tool presented here is mechanically the same as the one developed by Brown et al. [4, 6]; however addition of the sensing circuit board to the grasper surface requires recalibrating the jaw position and grasping force measurements.

The new tool includes sensors for measuring the temperature, local optical absorption spectrum, and electrical impedance of the grasped tissue packed into a $7 \mathrm{~mm}$ by $7 \mathrm{~mm}$ sensor head. Figure 5 shows a photograph of the sensor head and a photograph of the conditioning and control hardware and an operator display mounted in a portable box.

\subsection{Requirements}

There are many requirements that the design of the tool and sensors must satisfy. First, the sensors must align with the desired sensing modalities: tissue temperature, optical absorption spectrum, and electrical impedance and grasper jaw position and applied force. The sensors need to be tightly integrated on to the jaws of the grasper, preserving the functionality of the grasper for testing in vivo. The signal conditioning electronics and the operator interface hardware must be portable and robust to movement because the in vivo tests will take place in an operating room.

The data acquisition needs to occur fast enough to capture the heart beat and also have enough storage space to capture data for the length of the operation. In addition, the control system needs a fast enough update rate so that the tool motion is smooth and does not cause incidental damage to the tissue.

In addition, it is desirable to have the sensors and electronics that mount to the grasper jaws to be cheap for an ultimately disposable tool.

\subsection{Sensors and actuators}

The grasper body contains sensors for measuring the angle between the jaws and the applied force as well as an actuator for controlling either the jaw angle or applied force. The sensor head contains sensors for measuring tissue temperature, optical absorption, and electrical impedance.

The grasper's opening and closing is controlled by a brushed DC motor (RE25, $10 \mathrm{~W}$, Maxon). The angular position of the motor is sensed by an 500-count optical encoder (HEDL55, Maxon) on the motor shaft. The motor drives a 19:1 planetary gearbox (GP26, Maxon) that is connected to a capstan, which drives a 
partial pulley by means of a wire cable. A pushrod connects the partial pulley to the grasper jaws. The total mechanical advantage of this setup is $190: 1$.

The force applied by the jaws is sensed by a pair of strain gauges (FR1010, $40 \mathrm{lb}$, FUTEK Advanced Sensor Technology) mounted in the partial pulley in a double-beam configuration to eliminate bending moment. Due to the geometry of the partial pulley and the pushrod-to-jaw linkage, the relationship between the force sensed by the strain gauge and the force actually applied by the grasper jaws is dependent on the angle of the jaws.

The tool can apply approximately $26.5 \mathrm{~N}$ of grasping force at the maximum continuous motor torque of $29 \mathrm{mNm}$. This is significantly greater than the average force typically applied by surgeons to the tissue, $8.52 \mathrm{~N} \pm 2.77 \mathrm{~N}$ [5]. The tool is controlled to keep the applied force constant during a grasp to create a uniform contact interface.

The sensor head is mounted on the jaw surface and is visible in Fig. 5 (left). A thermistor (QT06002532, Quality Thermistor) measures the temperatures between $20^{\circ} \mathrm{C}$ and $47^{\circ} \mathrm{C}$.

A photodiode (PDB-C160SM, Advanced Photonix) on one of the grasper jaws and four LEDs on the opposite jaw form the optical spectroscopy system. The LED colors are infrared (SMC810, $810 \mathrm{~nm}$, Epitex), red (LTST-C190CKT, $660 \mathrm{~nm}$, Lite-On Electronics), green (LTST-C190KGKT, $574 \mathrm{~nm}$, Lite-On), and blue (LTST-C190TBKT, $468 \mathrm{~nm}$, Lite-On). The infrared and red wavelengths were chosen to match those used in pulse oximetry. With red, green, and blue LEDs, we can approximate the visible spectrum. There is also a photodiode (PDB-C152SM, Advanced Photonix) in the center of the four LEDs to measure reflected light. An ultraviolet LED (UVTOP335, $335 \mathrm{~nm}$, Sensor Electronic Technology) can be added to the array to measure fluorescence.

Four gold plated electrodes (4689-0-00-15-00-0033-0, Mill-Max Mfg. Corp.) linearly placed on the outer edge of the jaw provide electrical impedance sensing. The electrodes are $1.1 \mathrm{~mm}$ in diameter and spaced $2.6 \mathrm{~mm}$ apart.

The sensor head and opposing photodiode are encapsulated in clear epoxy (SB-112, System Three) to provide electrical isolation from the tissue. The electrodes are mounted so only the electrode surface comes into contact with the tissue. Silicone adhesive (RTV 162, General Electric) mounts the sensor head to the grasper.

\subsection{Analog electronics}

Each of the sensor outputs need signal conditioning before it can be sampled at $1 \mathrm{kHz}$. Signal conditioning and sampling is performed on a custom USB 2.0-based data acquisition board. The board supports up to eight quadrature inputs and 8 analog inputs $(0-2.56 \mathrm{~V}, 10$-bit resolution) and drive 8 analog outputs $( \pm 10 \mathrm{~V}, 16$-bit resolution). The board has been used in other projects in the BioRobotics Lab $[25,26]$. The signal conditioning circuits and data acquisition board are shown in Fig. 6.

The motor is driven by a linear four-quadrant servoamplifier (LSC 30/2, Maxon). The strain gauge signal is amplified by a stand-alone strain gauge amplifier (JM-2, FUTEK).

A wheatstone bridge and an op-amp buffer (INA128U, Burr-Brown) measure the thermistor resistance. The thermistor has a thermal time constant of $400 \mathrm{~ms}$, and the buffer output is low-pass filtered at $16 \mathrm{~Hz}$ to remove noise.

The brightness of each LED is individually controlled by adjustable current sources connected to analog outputs on the data acquisition board. The LEDs are time-multiplexed so that only one is emitting light at a given time, with each LED illuminated for $1 \mathrm{~ms}$ of the $6 \mathrm{~ms}$ cycle. The remaining $1 \mathrm{~ms}$ is used to measure the ambient light level.

The photodiodes are connected to transimpedance amplifiers (AD549, Analog Devices; OPA128, BurrBrown). The transimpedance amplifier feeds an inverting buffer (LM324, National Semiconductor) with an adjustable reference voltage. The adjustable reference voltage can be set to cancel out the low frequency response for each LED. This allows the buffer to function like a high-pass filter, which is often required to improve the signal to noise ratio for pulse oximetry.

A numerically controlled oscillator (AD9832, Ana$\log$ Devices) provides a sinusoidal reference output from $0 \mathrm{~Hz}$ to $25 \mathrm{MHz}$. A high speed amplifier (LM318D, National Semi-conductor) converts this to a current for the drive electrodes and the test tissue.

The voltage difference on the sense electrodes is amplified by an instrumentation amplifier (INA128U, Burr-Brown) and then compared to the reference output by a gain and phase detector (AD8302, Analog Devices). The detector provides the gain ratio up to $\pm 30 \mathrm{~dB}$ and phase difference up to $\pm 90^{\circ}$ between the two signals. 


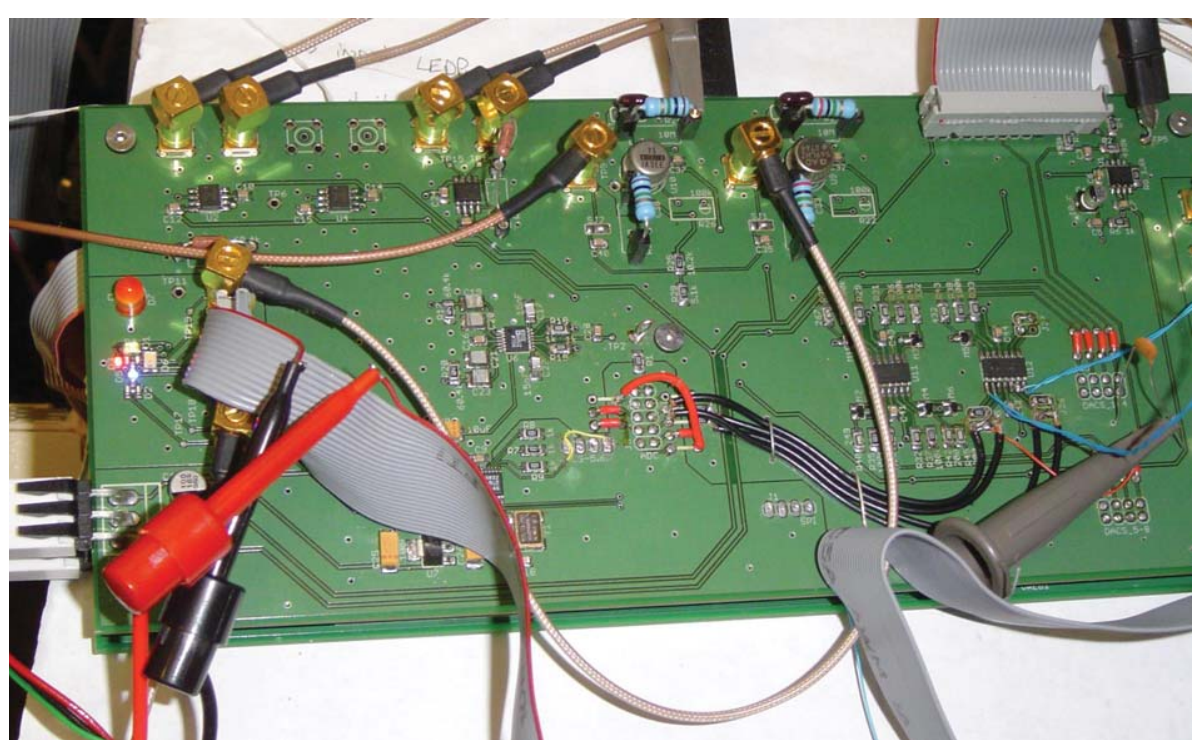

Fig. 6. A photograph of the analog signal conditioning board mounted to the data acquisition board.

\subsection{Real-Time software}

A general purpose computer running a version of the Linux operating system with Real-Time Application Interface (RTAI) extensions provides a real-time platform for handling the samples from the data acquisition board. The computer logs the received data and calculates the new outputs at $1 \mathrm{kHz}$. A proportionalintegral-derivative (PID) controller provides the motor output given the sensed force and the desired force. A fuzzy logic controller provides the LED outputs given the currently illuminated LED, the received photocurrent, and the desired brightness.

\subsection{Operator interface}

The real-time software pushes the calibrated data to the operator interface, which provides a graphical view of the data to the operator. Sensor readings are written to a $\log$ file and then displayed as strip charts with an adjustable history, which can show the most recent few seconds or the entire experiment. The graphical user interface (GUI) also provides touch screen controls for the operator: grasp force, grasp timing, desired LED brightness, impedance measurement frequencies, the data display rate. The GUI can be easily modified to display longer histories and provide more intuitive tool control for surgeons and engineers. The GUI can run locally over the RTAI code or it can run remotely over a network.

All of the hardware has been packaged into a briefcase-sized control box for convenient transportation to the operating room. A photograph of the control box is shown in Fig. 5. The control box includes a touchscreen and a few switches for easy operator interaction. In the operating room, the box is set up on a mobile cart near the patient. The grasper is mounted on a tripod to eliminate unnecessary sources of motion artifacts.

\section{Calibration}

The sensors need to be calibrated before they can provide useful measurements. The calibration goals were to measure the accuracy and repeatability of the sensors and to find the correlation between sensor readings and actual values. Each sensing modality was calibrated independently from the others.

These calibrations do not need to be performed before each experiment. However, in order to get accurate recordings, the zero position and zero grasp force values must be set before each experiment. The method for obtaining these values has been automated to achieve repeatable results. 


\subsection{Position}

The force measured by the strain gauge depends on the angle of the jaws. The angle of the jaws can be determined using the optical encoder, but due to compliance in the mechanism, the relationship between jaw angle and encoder also depends on the strain gauge reading.

The jaw angle was calibrated by squeezing multiple cardboard structures of various thicknesses with a range of forces, from $0.35 \mathrm{~N}$ to $7.0 \mathrm{~N}$. While this is well below the maximum force the tool can apply, it is sufficient for the initial tissue experiments. If the tool is required to grasp with forces outside this range, further calibration measurements will be required. Instead of measuring jaw angle directly, the separation distance of the jaws, $d_{\text {jaw }}$, is measured at the outermost end of the jaw, as shown in Fig. 7. The encoder counts, strain gauge reading, and measured distance are fit to a surface, providing an equation for calculating jaw position and angle.
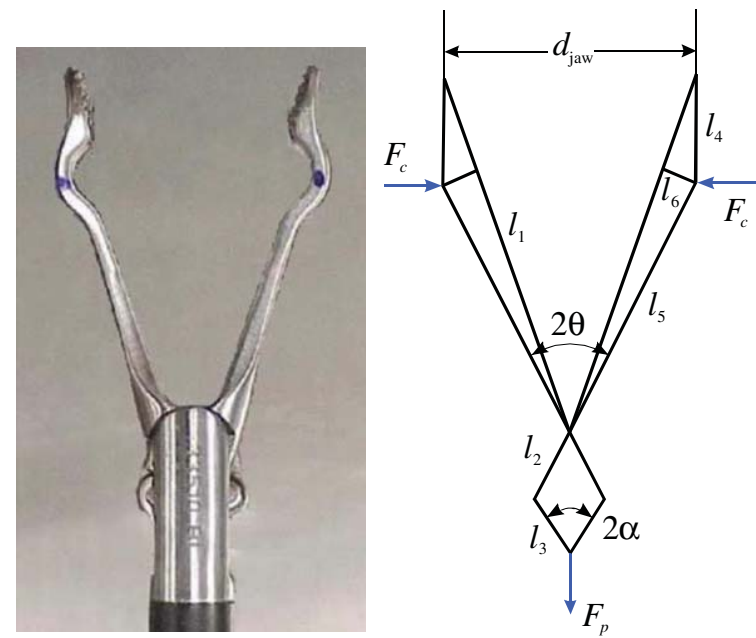

Fig. 7. (Left) A photograph of the grasper jaws without the sensor heads. The blue dots on the jaws indicate where the grasp force was measured by a digital force gauge. The tool measures force with a strain gauge at the other end of the tool shaft. (Right) A diagram of the mechanism with links and angles named. Equations 8 and 9 relate $F_{c}$ and $F_{p}$.

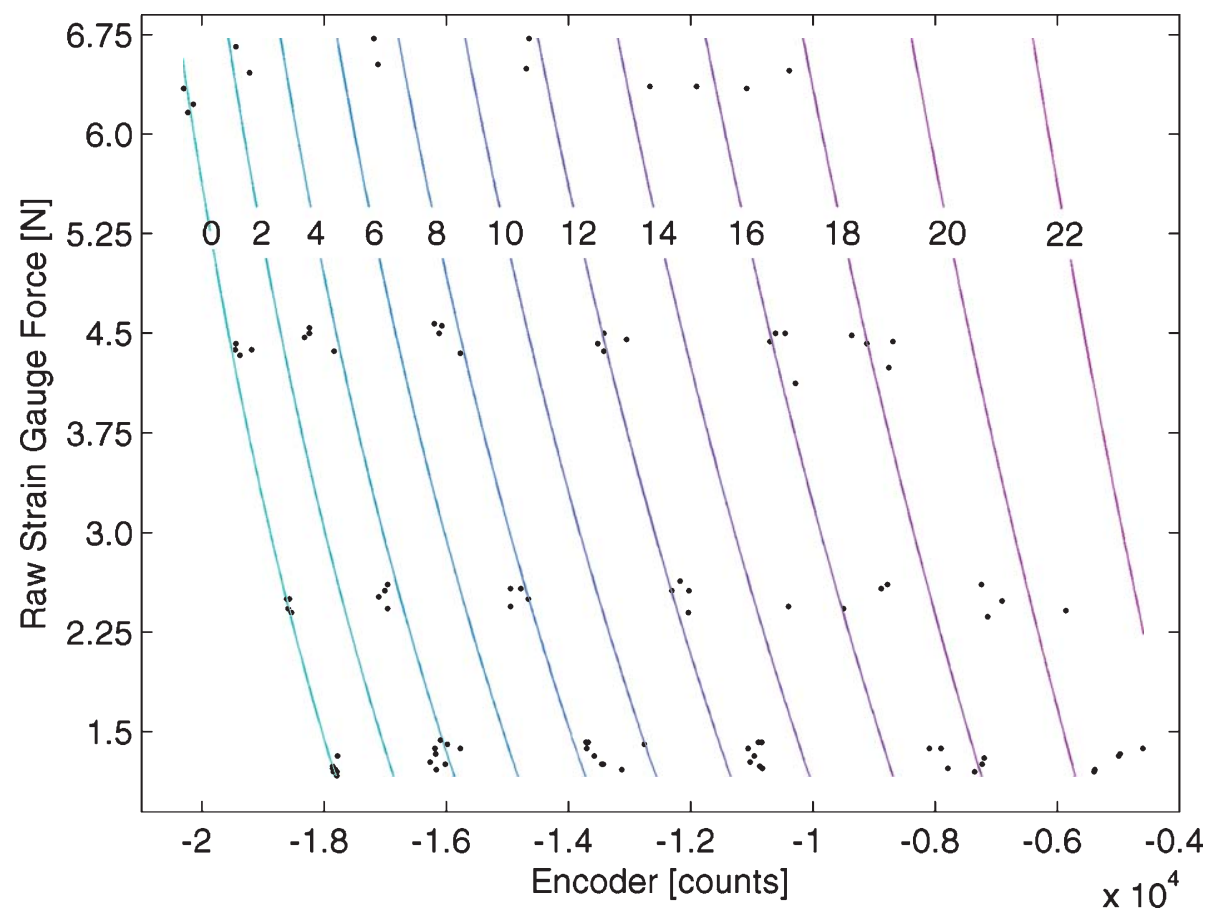

Fig. 8. Contour plot showing the actual position of the jaws as the measured force and encoder counts are varied. The contours give the position of the jaws in $\mathrm{mm}$. The difference between the contours and observed data points is due to the compliance of the cardboard structures. 
The data from the position calibration measurements are shown in Fig. 8 along with contours of the jaw position surface fit. The surface fit is described by

$$
d_{\mathrm{jaw}}=\frac{a+b x+c y+d x y}{1+e x+f y+g x y}+h
$$

where $x$ is the number of encoder counts from the jaws wide open and $y$ is the measured strain gauge force in bits. The constants $a, b, c, d, e, f, g$, and $h$ are given in Table 1 . The standard deviation of the data from the curve fit was found to be $0.283 \mathrm{~mm}$

The horizontal data clustering is due to the thickness of each cardboard sheet. The vertical data clustering is due to the discrete steps in applied force as seen in the strain gauge measurements. The contour lines clearly show the compliance in the mechanism. The clusters shift the left with increased force showing the compliance of the mechanism and the compliance of the cardboard.

\subsection{Force}

An apparatus was designed to allow the compressive force of the grasp to be measured with a digital force gauge (FG-5000, Extech Instruments). A pair of cables slip over the jaws and rest in the curve just inside of the grasper surfaces. One cable is fixed to a post and the other connects to the force gauge. The separation distance between the jaws was varied from $6 \mathrm{~mm}$ to $21 \mathrm{~mm}$ while forces up to $7.7 \mathrm{~N}$ were applied by the grasper. The forces were applied at discrete levels.

The relationship between force applied and force sensed can be calculated based on the linkage geometry. Figure 7 shows the links and their arrangement; Table 2 gives the link lengths. The blue dots on the grasper jaws in Fig. 7 (Left) show where the applied force, $F_{c}$, is measured by the digital force gauge.

Because the grasper surface is further away from the fulcrum than the measured force location, the force

Table 1

\begin{tabular}{lc} 
& Constants for the position fit \\
\hline$a$ & $-8.996 \cdot 10^{-1}$ \\
$b$ & $1.143 \cdot 10^{-3}$ \\
$c$ & $2.065 \cdot 10^{-3}$ \\
$d$ & $2.858 \cdot 10^{-7}$ \\
$e$ & $1.495 \cdot 10^{-5}$ \\
$f$ & $1.937 \cdot 10^{-3}$ \\
$g$ & $6.697 \cdot 10^{-8}$ \\
$h$ & $27.151 \mathrm{~mm}$ \\
\hline
\end{tabular}

Table 2

Grasper link lengths

\begin{tabular}{lr}
\hline$l_{1}$ & $32.44 \mathrm{~mm}$ \\
$l_{2}$ & $4.98 \mathrm{~mm}$ \\
$l_{3}$ & $4.90 \mathrm{~mm}$ \\
$l_{4}$ & $10.67 \mathrm{~mm}$ \\
$l_{5}$ & $22.13 \mathrm{~mm}$ \\
$l_{6}$ & $2.27 \mathrm{~mm}$ \\
\hline
\end{tabular}

applied over the grasper surface will be slightly less than $F_{c}$. The Babcock grasper used in the tool is designed to have uniform pressure over the grasping surface. This pressure is given by

$$
P_{s}=\frac{l_{1}-l_{s}}{l_{5}} F_{c} \cdot A s^{-1}
$$

where $l_{s}$ is the distance from the grasper tip to the center of the jaw surface, and $A_{s}$ is the area of the grasper surface in contact with the tissue.

The force in the pushrod, $F_{p}$, is proportional to the force measured by the strain gauge. The nonlinear relationship between $F_{c}$ and $F_{p}$ is

$$
\begin{aligned}
& F_{c}=F_{p} \cdot f_{\mathrm{jd}}(\theta) \\
& F_{p}=F_{c} \cdot f_{\mathrm{jd}}{ }^{-1}(\theta)
\end{aligned}
$$

where $f_{\mathrm{jd}}$ is the jaw displacement factor. From the geometry of the mechanism,

$$
f_{\mathrm{jd}}(\theta)=\frac{l_{2}}{l_{5}}\left(\tan \alpha+\frac{1}{2} \tan \theta\right)
$$

where

$$
\alpha=\sin ^{-1}\left(\frac{l_{2}}{l_{3}} \cdot \theta\right)
$$

and $\theta$ is the jaw angle, which can be derived from the jaw position, $d_{\text {jaw }}$ :

$$
\theta=\sin ^{-1}\left(\frac{\frac{1}{2} d_{\mathrm{jaw}}}{l_{1}}\right)+\sin ^{-1}\left(\frac{l_{6}}{l_{5}}\right)
$$

Figure 9 shows the force measured by the digital force gauge and the force measured by the strain gauge. The left half of the figure shows the dependence on jaw position and the different jaw positions are listed in the legend. The right half shows the measurements after compensation by the inverse jaw displacement factor.

The original formulation of the jaw displacement factor (Equation 9) was adjusted because of probable 

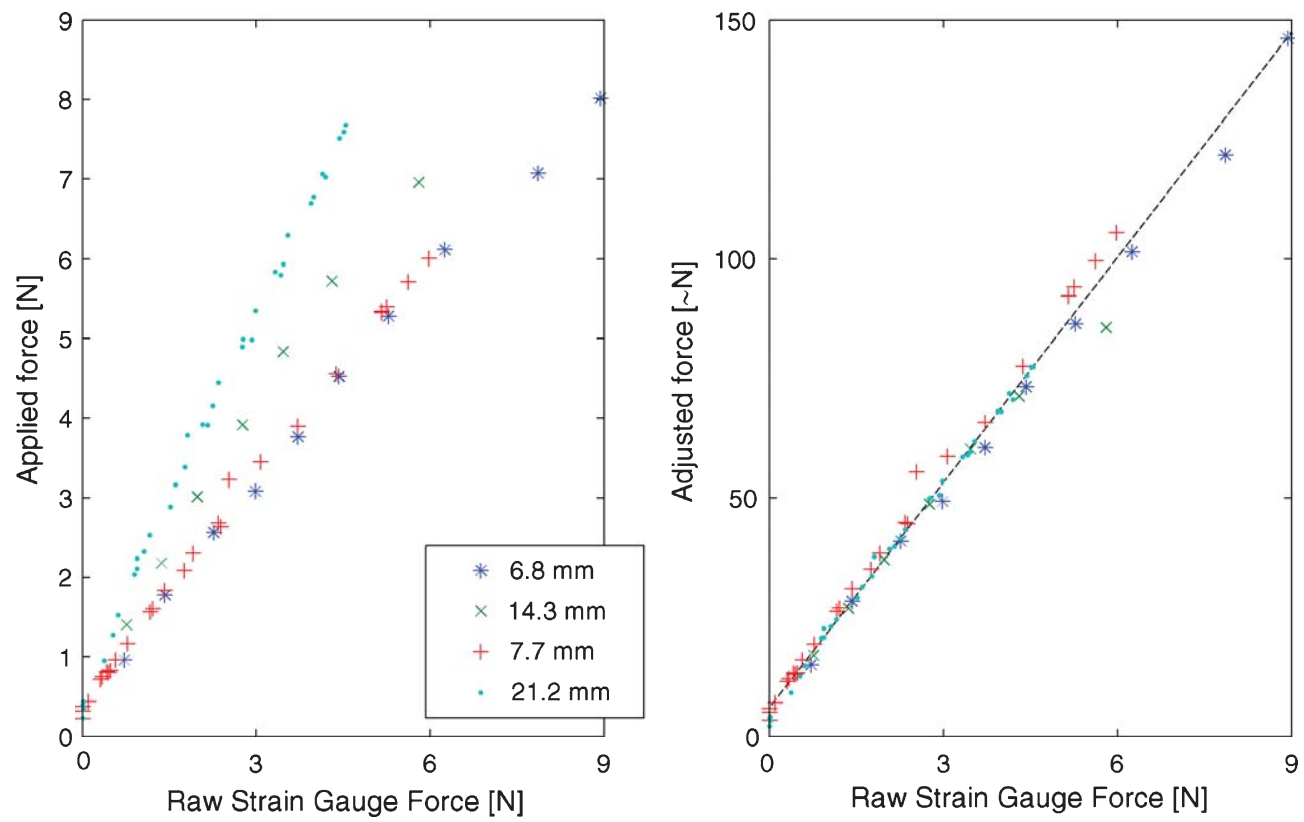

Fig. 9. (Left) The applied force as measured by the digital force gauge is plotted against the measured force. Each shape represents a different jaw angle. (Right) The transformed force plotted against the measured force showing how the dependence on jaw angle has been removed. The linear fit described by Equation 13 is also shown.

measurement errors in link length. The adjusted jaw displacement factor, $f_{\text {jda }}$ is related to $f_{\text {jd }}$ by

$$
f_{\mathrm{jda}}{ }^{-1}(\theta)=f_{\mathrm{jd}}{ }^{-1}(\theta)+3.656
$$

After calculating $F_{p}$, the linear relationship between $F_{p}$ and the force measured by the strain gauge, $F_{s}$ is given by

$$
F_{p}=0.236 \cdot F_{s}+6.007
$$

Figure 9 (right) shows this line. The standard deviation for the fit is 2.839 , which translates to $0.1616 \mathrm{~N}$ to $0.3042 \mathrm{~N}$ for applied force, depending on jaw angle.

\subsection{Temperature}

The thermistor was calibrated using a water bath. A hot plate maintained the bath at a $5^{\circ} \mathrm{C}$ increments from $20-50^{\circ} \mathrm{C}$ based on a thermocouple placed in the bath near the thermistor. The thermistor and thermocouple temperatures were recorded along with the temperature of the bath as measured by an infrared thermometer.

The Steinhart-Hart equation gives the relationship between temperature ( $T$, in kelvins) and resistance ( $R$, in ohms) for the thermistor:

$$
\frac{1}{T}=a+b \ln (R)+c \ln ^{3}(R)
$$

where $a, b$, and $c$ are thermistor specific constants listed in Table 3.

The temperature measured by the thermocouple and the thermistor were very closely related. A plot of the thermistor measurements, $T_{m}$, versus the thermocouple measurements, $T_{c}$, is shown in Fig. 10. The equation for the linear fit is

$$
T_{m}=1.0005 \cdot T_{c}+3.234
$$

The standard deviation for the temperature measurement is $0.09^{\circ} \mathrm{C}$.

\subsection{Optical spectroscopy}

Photocurrent produced by the photodiodes is a function of spectral irradiance of the light. Irradiance is the power per unit area of light incident on a surface, and

Table 3

Steinhart-Hart constants for the thermistor

\begin{tabular}{ll}
\hline$a$ & $1.145 \cdot 10^{-3}$ \\
$b$ & $2.314 \cdot 10^{-4}$ \\
$c$ & $9.943 \cdot 10^{-8}$ \\
\hline
\end{tabular}




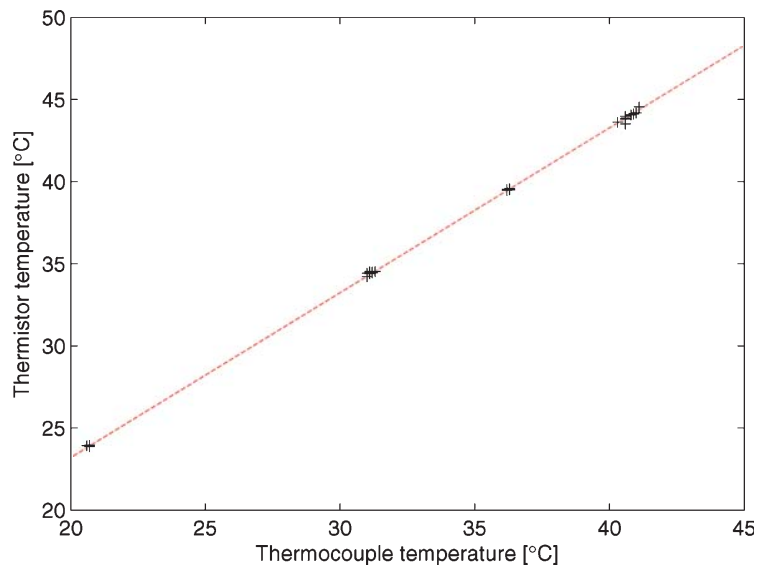

Fig. 10. Thermistor temperature versus thermocouple temperature. The linear fit described by Equation 15 is also shown.

spectral irradiance is wavelength dependent irradiance. The spectral irradiance on the photodiode is related to the brightness of the LED, the angle and distance between the photodiode and the LED, and the filtering effects of the tissue.

Photodiode responsivity describes the photocurrent for a given spectral irradiance. The photodiode responsivity curve provided by the manufacturer was verified with lasers at three discrete wavelengths: red $(660 \mathrm{~nm})$, green $(532 \mathrm{~nm})$, and blue $(405 \mathrm{~nm})$. Laser radiant flux and photocurrent were measured. The beam diameter of each laser was less than the edge length of the photodiode. The brightness of each LED was measured using an integrating sphere and a spectrophotometer. LED spectra were recorded at 8 to 15 forward currents from $100 \mu \mathrm{A}$ to $50 \mathrm{~mA}$.

The sensor head is enclosed in a black box for measuring the jaw angle dependence of the irradiance. Photocurrent and LED forward current are recorded for the full range of jaw distances $(0.0-26.2 \mathrm{~mm})$. An optical correction factor, $f_{\mathrm{PD}}\left(d_{\mathrm{jaw}}\right)$, is derived from this data.

The photocurrent, $i_{\mathrm{ph}}$, generated by the photodiode can be calculated by the integral

$$
i_{\mathrm{ph}}=\int S(\lambda) \cdot F(\lambda) \cdot D(\lambda) d \lambda
$$

where $S(\lambda)$ is the spectral radiant flux of the source, $F(\lambda)$ is the filter transmission spectrum, and $D(\lambda)$ is the responsivity of the detector. If $F(\lambda)$ is assumed to be constant over small ranges of wavelengths, then

$$
i_{\mathrm{ph}}=S\left(\lambda_{1}\right) \cdot F\left(\lambda_{1}\right) \cdot D\left(\lambda_{1}\right)
$$

Because the bandwidth of an LED is moderately narrow ( $15 \mathrm{~nm}-35 \mathrm{~nm}$ for the LEDs used on the tool), this assumption needs to be carefully considered. Knowing the approximate shape of the filter response over this bandwidth increases the accuracy as well.

If $S(\lambda)$ and $D(\lambda)$ are known at $\lambda_{1}$, the ideal photocurrent, $i_{\mathrm{ph}, \text { ideal }}$, can be calculated:

$$
i_{\text {ph,ideal }}=S\left(\lambda_{1}\right) \cdot D\left(\lambda_{1}\right)
$$

$F$ can be approximated by the ratio of $i_{\text {ph,ideal }}$ to the measured photocurrent, $i_{\text {ph,meas }}$ :

$$
F\left(\lambda_{1}\right)=\frac{i_{\text {ph,measured }}}{i_{\text {ph,ideal }}}
$$

Figure 11 shows the spectral radiant flux for the four LEDs. The spectral radiant flux is a measure of the LED brightness with units $\mathrm{W} \cdot \mathrm{nm}^{-1}$. Each contour line corresponds to a different LED forward current, with larger currents resulting in taller and wider peaks. The peaks for the infrared LED are much taller than those of the other colors but have been cut off in the figure for clarity of the other LEDs. The green LED is not very bright even at its rated maximum forward current. Spectra corresponding to unmeasured forward currents can be interpolated from these results.

The responsivity curve for the photodiode and the validation measurements are shown in Fig. 12. The measured values lie very close to the provided curve, and the deviations can likely be attributed to slight misalignment of the lasers.

The optical correction factor, $f_{\mathrm{PD}}\left(d_{\text {jaw }}\right)$ for each LED is shown in Fig. 13. The standard deviations are given as a percentage of the correction factor value at $12.7 \mathrm{~mm}$. The deviations are $11.73 \%$ for infrared, $1.98 \%$ for red, $1.68 \%$ for green, and $2.17 \%$ for blue.

With the basic optical absorption system calibrated, it can be used to measure tissue absorption and estimate $\mathrm{So}_{2}$. Further details are discussed in the following Section 5.

\subsection{Electrical impedance}

The impedance measurement system requires calibrating the current output circuits and the voltage input amplification circuits. The gain and phase detector chip (see Section 3.3) compares the reference signal to the amplified signal from the electrodes. The reference sig- 


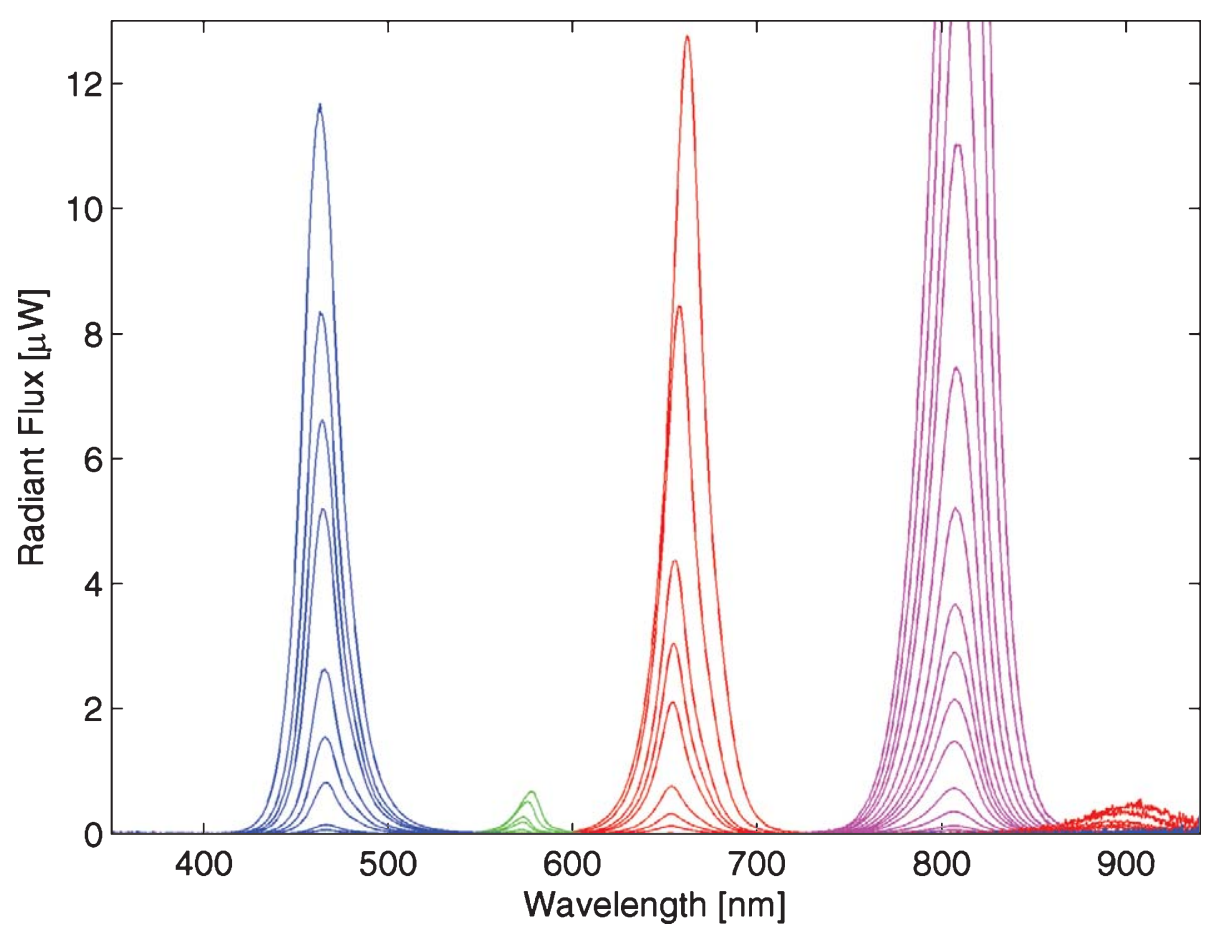

Fig. 11. Spectral radiant flux over a range of LED forward currents. The larger forward currents result in taller and wider peaks. The center wavelengths are $468 \mathrm{~nm}$ (blue), $574 \mathrm{~nm}$ (green), $660 \mathrm{~nm}$ (red), and $810 \mathrm{~nm}$ (infrared). The green LED has significantly lower radiant flux than the others, and the red LED has a small, secondary emission centered at $900 \mathrm{~nm}$.

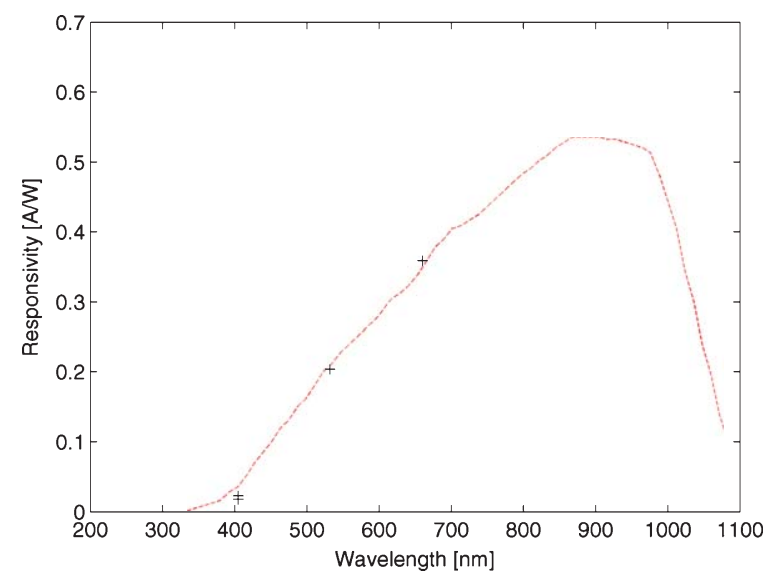

Fig. 12. Measured values of photodiode responsivity match well to the supplied responsivity curve.

nal magnitude and phase were measured at the detector input at frequencies from $1 \mathrm{kHz}$ to $5 \mathrm{MHz}$.

To calibrate the gain and phase detector, the system measured the impedance of five R-C networks (in the

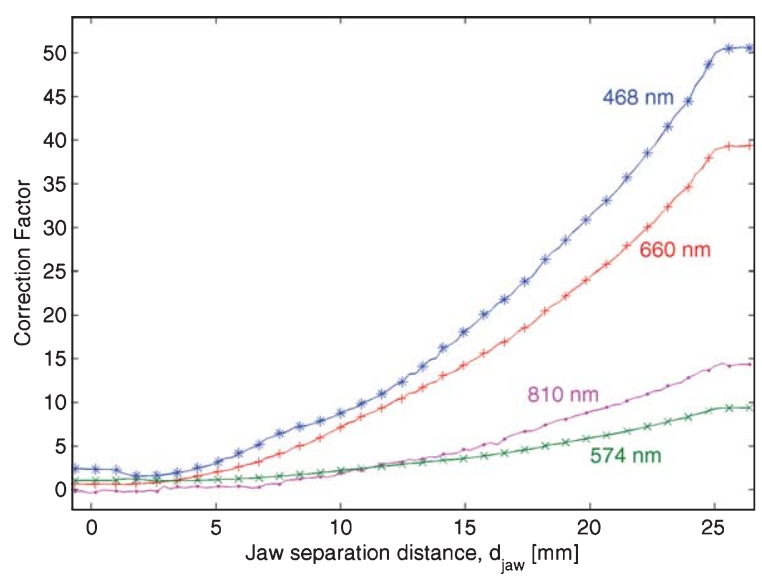

Fig. 13. The optical correction factor showing the jaw position dependence on the percentage of light received by the photodiode.

form shown in Fig. 3). The values for these models were adapted from [32] with $Z_{A}$ replaced by a capacitor, $C_{A}$. Resistor and capacitor values are shown in Table 4. 
Table 4

R-C network tissue model parameters

\begin{tabular}{lcrr}
\hline Tissue modeled & $\mathrm{R}_{\infty}$ & $\mathrm{R}_{0}-\mathrm{R}_{\infty}$ & $\mathrm{C}_{A}$ \\
\hline Liver & $540 \Omega$ & $1600 \Omega$ & $100 \mathrm{pF}$ \\
Lung & $417 \Omega$ & $1980 \Omega$ & $100 \mathrm{pF}$ \\
Spleen & $560 \Omega$ & $270 \Omega$ & $1.07 \mathrm{nF}$ \\
Muscle I & $254 \Omega$ & $97 \Omega$ & $17 \mathrm{nF}$ \\
Muscle II & $628 \Omega$ & $150 \Omega$ & $8.6 \mathrm{nF}$ \\
\hline
\end{tabular}

The reference signal magnitude and phase shift are shown in Fig. 14 along with the output from a SPICE simulation of the circuit. The magnitude starts to roll off at $500 \mathrm{kHz}$ due to a small amount of capacitance on the high speed op-amp, but it still remains well within the input range of the gain and phase detector. The $-3 \mathrm{~dB}$ cutoff frequency is approximately $1.9 \mathrm{MHz}$. The high speed op-amp inverts the signal, resulting in the $180^{\circ}$ phase shift. The phase shift is referenced to the signal coming from the numerically controlled oscillator.

Figure 15 shows the magnitude and phase outputs from the gain and phase detector chip for the R-C model of the spleen. The measured output exhibits similar behavior to the model, only with the break fre- quency occurring about a half-decade lower. This is likely due to parasitic capacitances that have not been included in the SPICE model. The outputs from the other R-C tissue models have similar differences from the SPICE model. A curve fit has not been calculated yet for this model because of the obvious shortcomings.

\section{Discussion}

The design and calibration of this tool indicate that it should be able to replicate the measurements described in Section 2. The design incorporates sensors able to measure the tissue temperature, optical absorption spectrum at four wavelengths, and electrical impedance. The chosen sensors are not the only sensors for measuring these modalities.

\subsection{Design}

The force applied by the grasper jaws is measured by a strain gauge coupled to the jaws through a linkage,
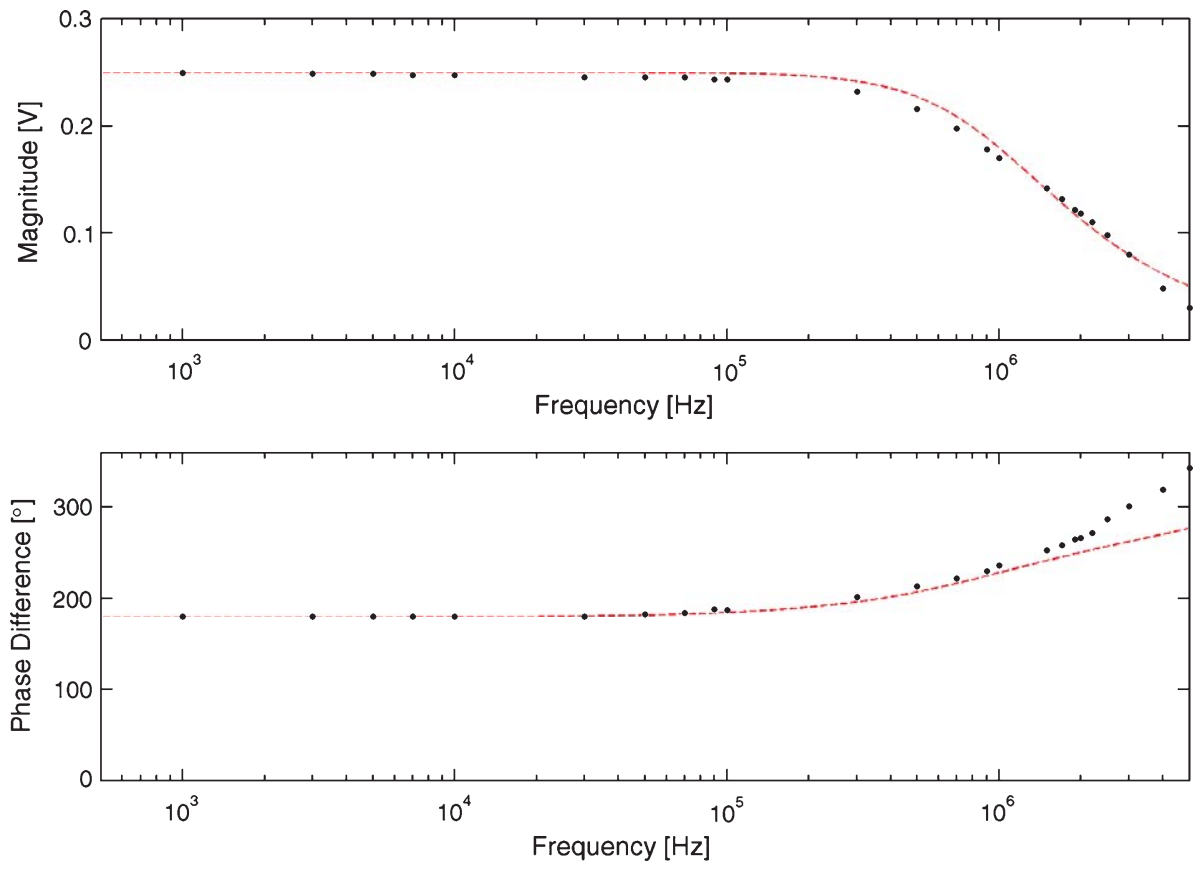

Fig. 14. Electrical impedance reference signal magnitude and phase shift over the range of operating frequencies. The signal is measured at the input to the gain and phase detector. 

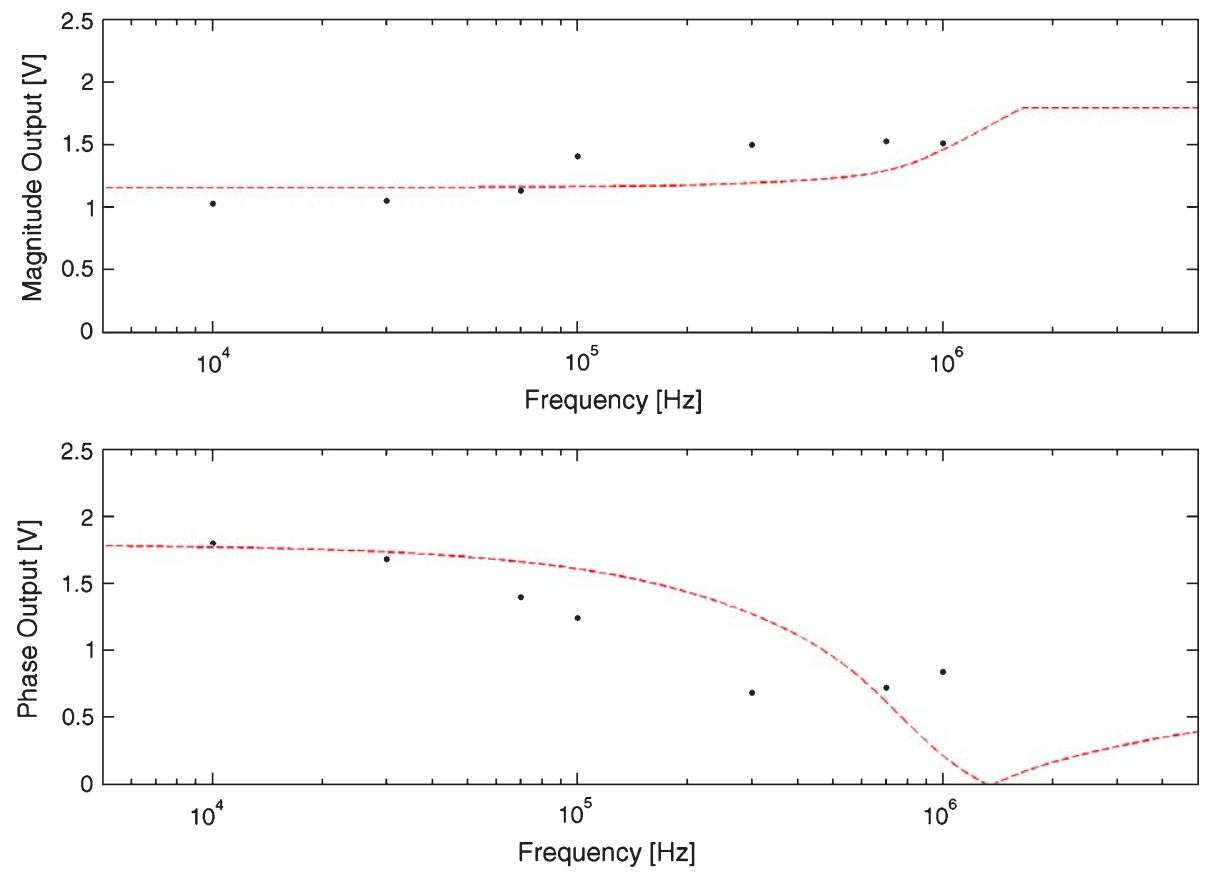

Fig. 15. Magnitude and phase outputs for the R-C model of the spleen. The expected behavior from the SPICE model is also plotted. The measured and modeled behaviors are similar except for a half-decade difference, which indicates an incomplete model.

where the friction and backlash can decrease the measurement accuracy. However, this location provides a reduction in noise as the signal from the strain gauges is very small and easily masked by electronic noise in the operating room.

Although using fiber optics and a spectrophotometer would provide a complete optical absorption spectrum, fibers change their transmission properties as they bend. LEDs and photodiodes were chosen because the LEDs are able to achieve similar results and are small, cheap, driven by a pair of wires. Future versions may incorporate optical fibers for spectroscopy.

\subsection{Calibration results}

The position and force sensors have been calibrated within the operating ranges of the tool. The contours in Fig. 8 show the compliance in the mechanism. Figure 9 (Left) shows how the applied force varies with jaw angle, but as can be seen in Fig. 9 (Right), f jda corrects this dependence.

The thermistor provides a precise measurement which will be useful for detecting changes in tissue temperature.
The filtering effects of the tissue will not be exactly like the effects of the color filters used in calibration. Optically dissimilar materials in tissue like blood, fat, muscle, and other tissue cells absorb light, and they reflect and diffuse light. These reflections can reach the photodetector, creating problems if the absorbance properties are being calculated solely with Beer's law (see Equation 1). However, proper modeling of these effects is very difficult $[9,45]$. Therefore, critical optical measurements of tissue properties, such as $\mathrm{So}_{2}$ will require calibration curves for each of the LEDs. Work is currently underway to generate the curves for this second level of tool calibration.

However, for diagnosing ischemia, the absolute level of blood oxygen saturation may not be critical information. It may be sufficient to observe a change or drop in a rough estimate of $\mathrm{So}_{2}$ during the procedure. This estimate would not require such extensive calibration curves, but it does require multiple measurements at the same site over the duration of the procedure. Alternatively, the tool can be placed on a section of healthy tissue to gather initial estimates of the absorption properties. This data can then be used as a patient specific calibration, and additional measurements during the 
operation can be compared against the initial, healthy measurement. Work is currently underway to validate these hypotheses.

There has been some previous work in the BioRobotics Lab on correlating tissue damage to tissue color $[10,11]$. This approach does not try to find the oxygen saturation of the tissue and therefore bypasses the approximations in that calculation. The instrumented tool described here can make rough measurements of tissue color and may be able to approximate tissue damage this way.

In design, the impedance measurement system was expected to measure a range of frequencies from $10 \mathrm{~Hz}$ to $10 \mathrm{MHz}$. However, the dynamic range of the signals over this range has proved to be too large. Additionally, the sense electrode voltage amplifier rolls off significantly for frequencies above $1 \mathrm{MHz}$. Performance could be improved by choosing a new amplifier with variable gain. The solution chosen is to restrict the range of frequencies to a more manageable $10 \mathrm{kHz}$ to $1 \mathrm{MHz}$.

Until the accuracy of the SPICE model is improved, tissue impedance cannot be accurately determined. Improvements include accounting for parasitic capacitances in the measurement leads and fully implementing the gain and phase detection.

\subsection{Potential benefits}

An instrumented tool for minimally invasive surgery can potentially offer benefits beyond monitoring tissue ischemia in real-time. For example, a sense of touch is beneficial to a surgeon as tissue diagnostic information can be acquired through palpation including tissue differentiation [20].

A single thermistor probe can measure temperature and simultaneously add heat for measuring thermal conductivity. The thermal conductivity of tissue can indicate the blood flow [13].

Individual tissues such as liver, spleen, and bladder can be differentiated based on their impedance profiles [17]. Additionally, impedance measurements taken at frequencies between $100 \mathrm{~Hz}$ and $1 \mathrm{MHz}$ were able to differentiate between normal, ischemic, and scar tissue [8]. Similarly, differences in tissue conductivity between $10 \mathrm{~Hz}$ and $1 \mathrm{MHz}$ can differentiate between normal and neoplastic tissue [19].

\section{Conclusions}

An instrumented tool for minimally invasive surgery that can detect tissue properties in real-time can improve patient care by lowering the amount of damage to tissue and reducing the rate of hospital readmission. The tool described here has been designed to sense many tissue properties using multiple sensing modalities. The sensor's mounting maintains the tool's functionality as a minimally invasive tool. The design requirements have been satisfied, and the calibration results verify that the tool will accurately measure the tissue properties with the exception of electrical impedance.

Electrical impedance cannot be accurately measured at this time. The different R-C tissue models can be differentiated, but the measured results do not match the expected results. Further refinements to the circuit should resolve this issue.

\subsection{Broader medical impacts}

This tool can be used to investigate the detection of tissue ischemia and damage in real-time. Those results will affect the integration of technology into surgery and medicine. It will help determine if instrumented tools will be useful to the surgeon. It may be the case that the signal to noise ratio of this type of sensor is too low to make a useful tool.

The tool could provide feedback (tactile, visual, aural) to the surgeon when the tool is causing significant tissue damage or the tissue is less than optimal because it is too dense or does not have enough blood flow. Additionally, the tool has the potential to diagnose cancerous tissue and determine tumor margins in real-time.

In general, an instrumented surgical tool is another step towards bringing science into the operating room to improve the patient standard of care. In the future, this type of tool will be useful in enhancing remote surgery and enabling automated surgery.

\section{References}

[1] D.A. Benaron, I.H. Parachikov, W.F. Cheong, S. Friedland, B. Rubinsky, D.M. Otten, F.W.H. Liu, C.J. Levinson, A.L. Murphy, J.W. Price, et al., Design of a visible-light spectroscopy clinical tissue oximeter, Journal of Biomedical Optics 10(4) (2005), 044005. 
[2] A. Bozkurt and B. Onaral, Safety assessment of near infrared light emitting diodes for diffuse optical measurements, BioMedical Engineering OnLine 3(1) (2004), 9.

[3] J.P. Brooks, W.B. Perry, A.T. Putnam and R.E. Karulf, Thermal imaging in the detection of bowel ischemia, Diseases of the Colon and Rectum 43(9) (2000), 1319-1321.

[4] J.D. Brown, J. Rosen, Y.S. Kim, L. Chang, M. Sinanan and B. Hannaford, In-vivo and in-situ compressive properties of porcine abdominal soft tissues, Studies in Health Technology and Informatics - Medicine Meets Virtual Reality 94 (2003), 26-32.

[5] J. Brown, In-vivo and postmortem biomechanics of abdominal organs under compressive loads: experimental approach in a laparoscopic surgery setup. Ph.D. Thesis. 2003.

[6] J. Brown, J. Rosen, M. Moreyra, M. Sinanan and B. Hannaford, Computer-controlled motorized endoscopic grasper for in vivo measurements of soft tissue biomechanical characteristics, Studies in Health Technology and Informatics Medicine Meets Virtual Reality 85 (2002), 71-73.

[7] J.B. Bussemaker and J. Lindeman, Comparison of methods to determine viability of small intestine, Annals of Surgery 176(1) (1972), 97-101.

[8] O. Casas, R. Bragós, P. Rui, J. Rosell, M. Tresaǹchez, M. Warren, A. Rodriguez-Sinovas, A. Carrenõ and J. Cinca, In vivo and in situ ischemic tissue characterization using electrical impedance spectroscopy, Annals New York Academy of Sciences 873 (1999), 51-58.

[9] W.F. Cheong, S.A. Prahl and A.J. Welch, A review of the optical properties of biological tissues, IEEE Journal of Quantum Electronics 26(12) (1990), 2166-2185.

[10] S. De, A. Dagan, P. Roan, J. Rosen, M. Sinanan, M. Gupta and B. Hannaford, CIELab and sRGB color values of in vivo normal and grasped porcine liver, In: Proceedings MMVR 2007, Feb 6-9. Long Beach, CA, 2007.

[11] S. De, J. Rosen, A. Dagan, P. Swanson, M. Sinanan and B. Hannaford, Tissue damage due to mechanical stresses as applied during minimally invasive surgery, International Journal of Robotics Research 26 (2007), 1159-1171.

[12] S. De, P. Swanson, M. Sinanan, J. Rosen, A. Dagon and B. Hannaford, Tissue damage due to mechanical stresses as applied during minimally invasive surgery, In: Proceedings of the 2006 BioRob Conference, Feb. Pisa, Italy, 2006.

[13] G. Delhomme, W.H. Newman, B. Roussel, M. Jouvet, H.F. Bowman and A. Dittmar, Thermal diffusion probe and instrument system for tissue blood flow measurements: validation in phantoms and in vivo organs, IEEE Transactions on Biomedical Engineering 41(7) (1994), 656-662.

[14] G.S. Fischer, T. Akinbiyi, S. Saha, J. Zand, M. Talamini, M. Marohn and R. Taylor, Ischemia and force sensing surgical instruments for augmenting available surgeon information, In: International Conference on Biomedical Robotics and Biomechatronics, February, 2006.

[15] H. Fischer, B. Neisius and R. Trapp, Tactile feedback for endoscopic surgery. Interactive Technology and the New Paradigm for Health Care, 1995.

[16] S. Friedland, D.A. Benaron, S. Coogan, D.Y. Sze and R. Soetikno, Diagnosis of chronic mesenteric ischemia by visible light spectroscopy during endoscopy, Gastrointestinal Endoscopy 65(2) (2007), 294-300.

[17] S. Gabriel, R.W. Lau and C. Gabriel, The dielectric properties of biological tissues: II. Measurements in the frequency range
$10 \mathrm{~Hz}$ to $20 \mathrm{GHz}$, Physics in Medicine and Biology 41 (1996), 2251-2269.

[18] Gersing E, Impedance spectroscopy on living tissue for determination of the state of organs, Bioelectrochemistry and Bioenergetics 45 (1998), 145-149.

[19] D. Haemmerich, S. Staelin, J.Z. Tsai, S. Tungjitkusolmun, D.M. Mahvi and J.G. Webster, In vivo electrical conductivity of hepatic tumours, Physiological Measurement 24 (2003), 251-260.

[20] B. Hannaford, J. Trujillo, M. Sinanan, M. Moreyra, J. Rosen, J. Brown, R. Lueschke and M. MacFarlane, Computerized endoscopic surgical grasper, In: Proceedings, MMVR-98 (Medicine Meets Virtual Reality), San Diego, Jan 1998.

[21] R. Jennings, C. Ganote and K. Reimer, Ischemic tissue injury, The American Journal of Pathology 81(1) (1975), 179.

[22] A. Jubran, Pulse oximetry, Crit Care 3(2) (1999), R11 -R17.

[23] T.P. Kingham and H.L. Pachter, Colonic anastomotic leak: risk factors, diagnosis, and treatment, Journal of the American College of Surgeons 208(2) (2009), 269-278.

[24] S. Kun and R.A. Peura, Tissue ischemia detection using impedance spectroscopy, In: IEEE Engineering in Medicine and Biology Society 2 (1994), 868-869.

[25] R. Leuschke, E. Kurihara, J. Dosher and B. Hannaford, High Fidelity Multi Finger Haptic Display. Haptic Interfaces for Virtual Environment and Teleoperator Systems 2005. WHC 2005, pp. 606-608.

[26] M. Lum, D. Trimble, J. Rosen, H. King, G. Sankarayanaranan, J. Dosher, R. Leuschke, B. Martin-Anderson, M. Sinanan and B. Hannaford, Multidisciplinary approach for developing a new minimally invasive surgical robot system, In: Proceedings of the 2006 BioRob Conference, Feb. Pisa, Italy, 2006.

[27] E.T. McAdams and J. Jossinet, Tissue impedance: a historical overview, Physiological Measurement 16 (1995), A1-A13.

[28] A.K. Morimoto, R.D. Foral, J.L. Kuhlman, K.A. Zucker, M.J. Curet, T. Bocklage, T.I. MacFarlane and L. Kory, Force sensor for laparoscopic Babcock, Studies in Health Technology and Informatics 39 (1997), 354.

[29] M.S. Patterson, Chance B and B.C. Wilson, Time resolved reflectance and transmittance for the non-invasive measurement of tissue optical properties, Applied Optics 28(12) (1989), 2331-2336

[30] S.A. Prahl, Optical Absorption of Hemoglobin, 1999. http:// omlc.ogi.edu/spectra/hemoglobin/summary.html

[31] J.L. Reuss and D. Siker, The pulse in reflectance pulse oximetry: modeling and experimental studies, Journal of Clinical Monitoring and Computing 18(4) (2004), 289-299.

[32] B. Rigaudt, L. Hamzaouit, M.R. Frikhaf, N. Chauveaut and J.P. Moruccit, In vitro tissue characterization and modelling using electrical impedance measurements in the $100 \mathrm{~Hz}$ $10 \mathrm{MHz}$ frequency range, Physiological Measurement 16 (1995), A15-A28.

[33] B. Ristic, S. Kun and R.A. Peura, Muscle tissue ischemia monitoring using impedance spectroscopy: quantitative results of animal studies, In: IEEE Engineering in Medicine and Biology Society. vol. 19 November 1997, pp. 2108-2111.

[34] S. Romano, F. Lassandro, M. Scaglione, L. Romano, A. Rotondo and R. Grassi, Ischemia and infarction of the small bowel and colon: spectrum of imaging findings, Abdominal Imaging 31(3) (2006), 277-292.

[35] J. Rosen, B. Hannaford, M. MacFarlane and M. Sinanan, Force controlled and teleoperated endoscopic grasper for 
minimally invasive surgery - experimental performance evaluation, IEEE Transactions on Biomedical Engineering 46(10) (1999), 1212-1221.

[36] L.S. Rosenthal and L.J. Brandt, Intestinal Ischemia, 2007. http://www.acg.gi.org/patients/gihealth/ischemia.asp.

[37] F. Seoane, J. Ferreira, J.J. Sanchéz and R. Bragós, An analog front-end enables electrical impedance spectroscopy system on-chip for biomedical applications, Physiological Measurement 29 (2008), S267-S278.

[38] J.E. Sinex, Principles and limitations, The American Journal of Emergency Medicine 17(1) (1999), 59-67.

[39] B.R. Soller, N. Cingo, J.C. Puyana, T. Khan, C. Hsi, H. Kim, J. Favreau, S.O. Heard, Simultaneous measurement of hepatic tissue $\mathrm{pH}$, venous oxygen saturation and hemoglobin by near infrared spectroscopy, Shock 15(2) (2001), 106-111.

[40] P. Steendijk, G. Mur, E.T. van derVelde and J. Baan, The Four-electrode resistivity technique in anisotropic media: theoretical analysis and application on myocardial tissue in vivo, IEEE Transactions on Biomedical Engineering 40(11) (1993), 1138-1148.

[41] J.G. Webster, editor. Medical instrumentation: application and design, 4 ed, John Wiley and Sons, 2009.

[42] L. Yang, M. Egawa, M. Akimoto and M. Miyaka, An imaging colorimeter for noncontact skin color measurement, The Optical Society of Japan 10(6) (2003), 554-561.

[43] X. Yu, H.J. Chizeck and B. Hannaford, Comparison of transient performance in the control of soft tissue grasping, In: Proceedings of the IEEE/RSJ International Conference on Intelligent Robots and Systems, San Diego, CA, Oct, 2007a.

[44] X. Yu, H. Chizeck and B. Hannaford, Comparison of transient performance in the control of soft tissue grasping, In: Proceedings of IROS 2007, San Diego, CA, $2007 \mathrm{~b}$.

[45] G. Zonios, U. Shankar and V.K. Iyer, Pulse oximetry theory and calibration for low saturations, IEEE Transactions on Biomedical Engineering 51(5) (2004), 818-822. 

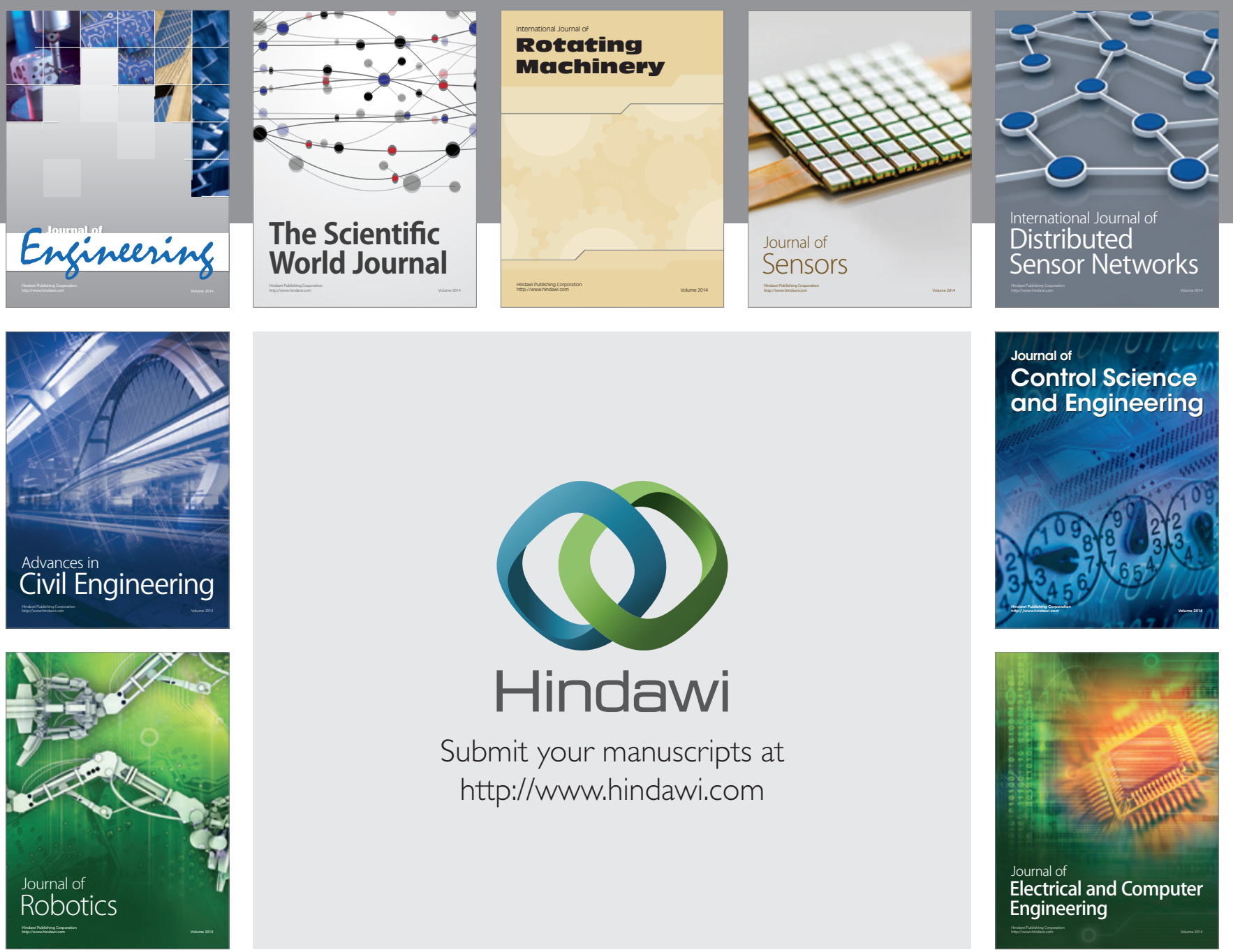

Submit your manuscripts at

http://www.hindawi.com
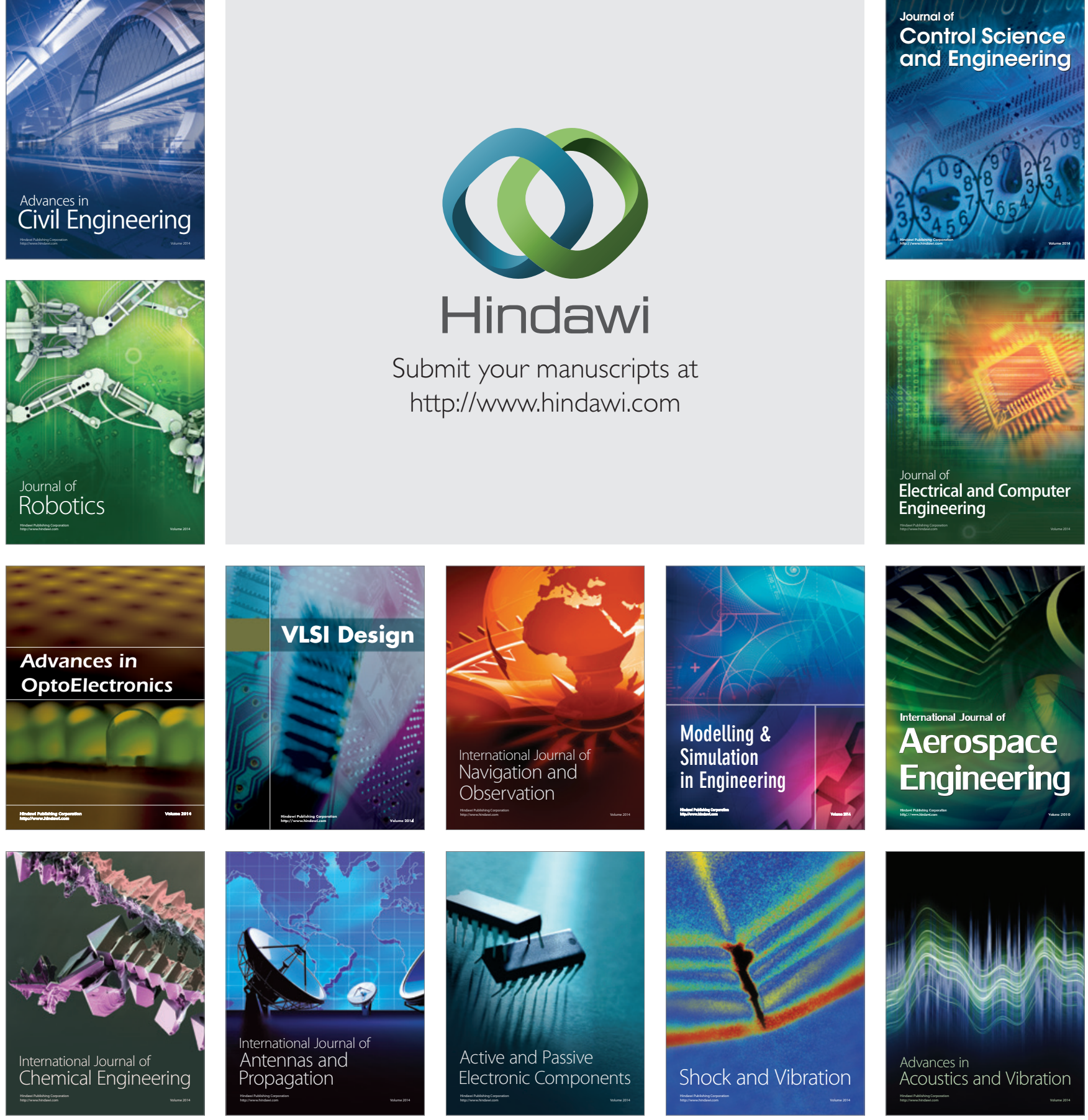